J3eA, Journal sur l'enseignement des sciences et technologies de l'information et des systèmes, Volume 4, Hors-Série 2, 10 (2005)

DOI : http://dx.doi.org/10.1051/bib-j3ea:2005710

(C) EDP Sciences, 2005

\title{
Mesures automatiques en électrotechnique
}

E. David, A. Dworak, F. Louboutin et G. Stevenard

Département de Physique Appliquée et de Génie Électrotechnique L.S.T. Colbert de Tourcoing, Parvis Jean-Baptiste Colbert

F-59200 Tourcoing, France 


\title{
MESURES AUTOMATIQUES EN ELECTROTECHNIQUE
}

\author{
E. DAVID, A . DWORAK, F. LOUBOUTIN, G. STEVENARD Département de Physique Appliquée et de \\ Génie Électrotechnique, L.S.T. COLBERT de TOURCOING, Parvis Jean Baptiste COLBERT, 59200 \\ TOURCOING. \\ Ce.0590214m@ac-lille.fr
}

Résumé : Le lycée COLBERT de TOURCOING est équipé d'un laboratoire d'électrotechnique disposant des équipements nécessaires à l'automatisation des travaux pratiques liés aux convertisseurs statiques et machines tournantes. mesures).

Le laboratoire, d'une superficie de $200 \mathrm{~m}^{2}$, permet d'accueillir quatorze étudiants (sept postes de

Dans l'objectif de recentrer un certain nombre de T.P. sur l'analyse, l'interprétation de résultats de mesures réalisées sur des équipements à caractère industriel, une automatisation importante est apportée aux procédures de mesures. Dans le souci de pouvoir adopter cette stratégie sur l'ensemble du programme des classes concernées, BTS électrotechnique et classe préparatoire TSI, l'équipe pédagogique a préféré l'utilisation d'une instrumentation électronique gérée par ordinateur, avec la possibilité de développer ses propres applications, plutôt que l'achat de cartes d'interfaces spécialisées avec logiciel spécifique.

Mots clés : mesures, automatiques, électrotechnique.

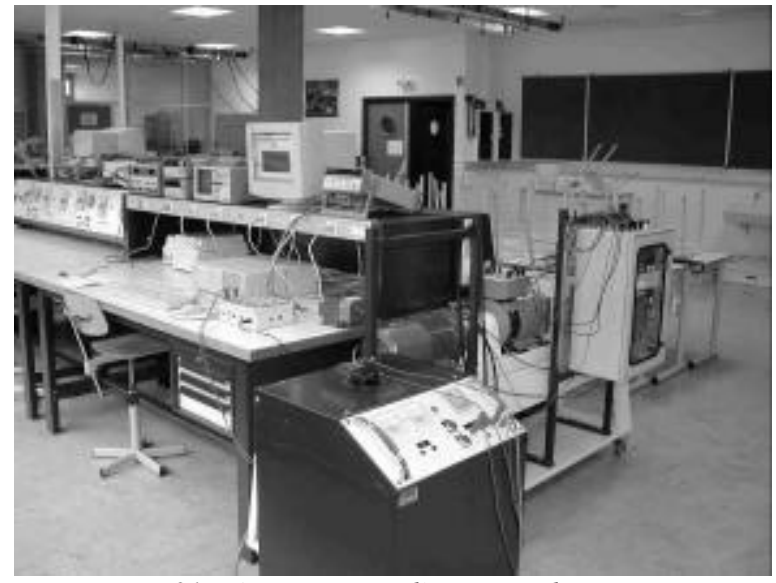

Fig $n^{\circ} 1$ : Organisation d’un poste de mesures

\section{Présentation du laboratoire d'électrotechnique}

1.1. Bancs de machines Les bancs de machines comportent deux machines tournantes accouplées à un volant d'inertie, une charge de type frein à poudre est souvent disponible. Pour la mesure des grandeurs mécaniques, chaque banc dispose de capteurs de mesures de position (codeur incrémental) et vitesse (génératrice tachymétrique), ainsi que de capteurs de force ou de couple.

\subsection{Variateurs de vitesse industriels Les} machines tournantes peuvent être alimentées par des variateurs industriels :

- Les machines à courant continu, de puissance $1.5 \mathrm{~kW}$, sont commandées par des variateurs quatre quadrants de type WNTC de la société CEGELEC (pont tout thyristor).
- Les moteurs asynchrones à cages, de puissance $1.5 \mathrm{~kW}$, sont commandés par des variateurs à contrôle vectoriel de flux, de type VFTV de la société CEGELEC.

Les deux types de variateurs WNTC et VFTV, dont la partie commande est de technologie entièrement numérique, sont paramétrés et commandés par un environnement PC, grâce à une interface RS232.

1.3. Instrumentation de mesures Les postes de manipulations sont équipés d'instruments de mesures automatisés, pilotés par un environnement PC, au travers d'un bus HP-IB. L'instrumentation est composée de :

- Un oscilloscope numérique HP54600B.

- Un générateur de fonctions, pouvant générer des signaux arbitraires, HP33120A.

- Une centrale d'acquisition 20 voies, permettant l'acquisition de toutes les grandeurs mécaniques et électriques, HP34970A. Le raccordement des signaux à la centrale est réalisé grâce un boîtier 
de conception « maison » comportant une électronique de conditionnement.

- Une alimentation stabilisée programmable, HP E3631A ou E3632A.

- Un multimètre numérique 200000 points, HP34401A.

1.4. Parc informatique Le laboratoire est équipé de huit micro-ordinateurs et d'une imprimante reliés en réseau. Le pilotage des manipulations, au travers du réseau, est possible.

1.5. Logiciel de gestions automatiques Le logiciel d'acquisition de données et pilotage automatique, actuellement utilisé, est HP-VEE 5.0. Les applications développées permettent :

- Le paramétrage et la commande des variateurs de vitesse.

- Le paramétrage de l'instrumentation et l'acquisition des données.

- Le traitement mathématique des enregistrements.

- La réalisation d'une interface graphique hommemachine pour les différentes applications développées.

- La gestion de manipulations au travers d'un réseau Ethernet dans une configuration clientserveur.

\section{Exemples de travaux pratiques automatisés}

\subsection{Pont tout thyristor, redressement commandé} monophasé Utilisation de la centrale d'acquisition pour le relevé des valeurs moyenne, efficace de l'ondulation, efficace vraie des grandeurs tensions et courants du montage. Utilisation de l'analyse spectrale du courant et de la tension côté continu, pour identification des paramètres résistif et inductif de la charge.

\subsection{Relevés de caractéristiques de machine à} courant continu Relevé automatique de la caractéristique à vide de la machine en fonctionnement génératrice. Etalonnage du capteur de couple par relevé de la tension différentielle du pont de jauge fonction du couple. Relevé automatique des caractéristiques électromécaniques du moteur.

\subsection{Relevés de caractéristiques de machine} synchrone Relevé automatique des caractéristiques à vide, en court-circuit et de réglage de la machine synchrone.

\subsection{Fonctionnement dynamique quatre} quadrants de la machine à courant continu Machine à courant continu, chargée d'un volant d'inertie, d'un frein à poudre, alimentée par un variateur WNTC en configuration variation de vitesse.

- Fonctionnement un quadrant, essai de Routin afin d'identifier le couple de pertes et l'inertie totale entraînée.

- Fonctionnement deux quadrants, freinage par récupération : mesures des accélération et décélération, mise en évidence des paramètres du variateur et des caractéristiques du banc de machines qui influencent ces grandeurs.

- Fonctionnement quatre quadrants, inversion du sens de rotation, paramétrage du variateur pour un profil de vitesse trapézoïdal donné.

\subsection{Asservissement de vitesse d'une machine à} courant continu Etude de la régulation cascade du variateur WNTC en configuration variation de vitesse.

Détermination des paramètres d'un modèle linéaire de la boucle de régulation de courant, exemples :

- Amplification apportée par le pont tout thyristor associé à son électronique de commande,

- Identification des composantes résistive et inductive de la charge présentée par l'induit de la machine à courant continu, etc ...

A l'aide du modèle linéaire de la boucle de courant, calcul des coefficients du correcteur numérique de la boucle de courant, paramètrage du variateur et essai indiciel sur la boucle de courant, rotor de la machine bloqué.

Analyse harmonique de la boucle ouverte de vitesse non corrigée et de la boucle fermée de vitesse. Calcul des coefficients du correcteur numérique de vitesse, paramétrage du variateur, nouvelle analyse harmonique pour vérifier la bande passante en boucle fermée ainsi obtenue.

2.6. Réversibilité des machines alternatives et continues La machine asynchrone est alimentée par le réseau industriel, la machine à courant continu par un variateur industriel commandé par une consigne de couple. Le T.P. est centré sur le relevé de la caractéristique couple utile fonction de la vitesse $\mathrm{Cu}=\mathrm{f}(\Omega)$, avec un fonctionnement dans deux quadrants de chaque machine. Pour analyser le comportement du banc, l'étudiant dispose de mesures de courant et de puissances. L'interprétation de relevés $\mathrm{Cu}$ $=\mathrm{f}(\Omega)$, pour plusieurs tensions d'alimentation de la machine asynchrone, est à réaliser.

3. Apport de la mesure automatique dans les travaux pratiques d'électrotechnique

«L'asservissement de vitesse d'une machine » à courant continu est le support qui illustrera l'apport de la mesure automatique dans la conduite de travaux pratiques en 
électrotechnique. Un schéma fonctionnel simplifié de l'asservissement est proposé en annexe $\mathrm{n}^{\circ} 1$, le repérage des grandeurs et des registres fait référence à ce document. L'étude de l'asservissement est réalisée en deux séances de 4 heures, une séquence pour la boucle de régulation de courant, une autre pour la boucle de vitesse.

3.1. Etude de la boucle de courant Un modèle linéaire de la boucle de courant est proposé (fig $\mathrm{n}^{\circ} 2$ ), la suite des expérimentations a pour but d'identifier un certain nombre de paramètres du schéma fonctionnel de la boucle ouverte.
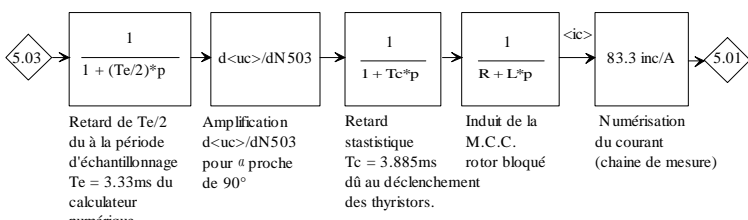
calculateur
umérique des thyristors.

Modélisation de la boucle ouverte de courant sans le correcteur numérique.

Fig $n^{\circ} 2$ : Boucle ouverte de courant non corrigée

(a) Un premier cycle de mesures automatiques permet l'identification de la loi liant la tension d'induit $\mathrm{U}_{\text {ind }}$ délivrée par le variateur WNTC au registre de commande du retard à l'amorçage des ponts tout thyristor (N503 contenu du registre 5.03).

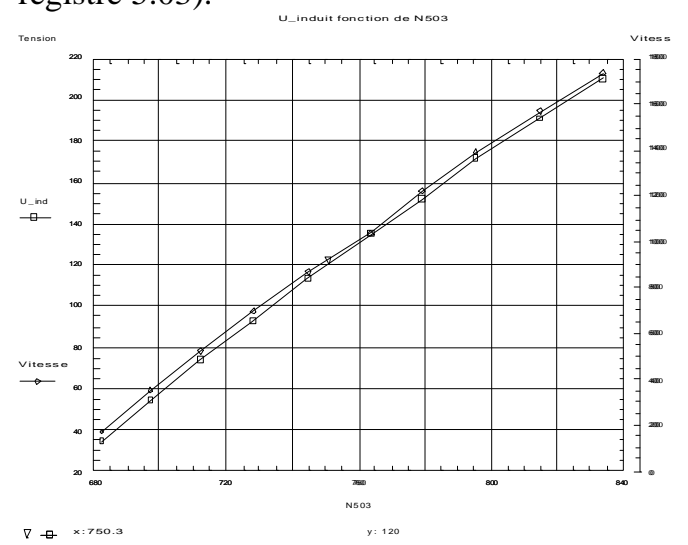

Fig $n^{\circ} 3$ : Vitesse et tension d'induit fonction de N503

Cette courbe est tracée par simple interrogation du variateur sur le contenu des registres 3.04 (valeur de la tension d'induit) et 5.03, pour dix consignes de vitesse différentes. Les courbes obtenues sont représentées sur la figure $n^{\circ} 3$, une régression linéaire sur les dix points permet d'identifier « l'amplification » apportée par le convertisseur alternatif continu associé à son électronique de commande. Les commentaires attendus dans la rédaction du compte-rendu portent sur le type de loi mathématique reliant l'angle électrique de retard à l'amorçage $\alpha$, au contenu du registre de commande 5.03.

(b) La mesure des composantes résistive et inductive de l'induit de la machine est obtenue par exploitation des analyses de FOURIER des oscillogrammes de la tension et du courant d'induit (fig n ${ }^{\circ}$ ), rotor bloqué. Cette technique de mesure des composantes d'une charge R-L est préalablement introduite dans la séquence «Pont tout thyristor, redressement commandé monophasé ».

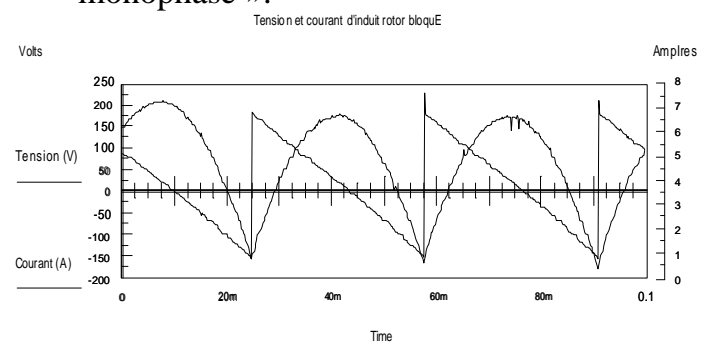

Fig $n^{\circ} 4$ : Tension et courant d'induit rotor bloqué

(c) Le modèle linéaire de la boucle de courant étant complété, la séquence se poursuit par la prédétermination des coefficients du correcteur : $\mathrm{K}_{\mathrm{pi}}+\mathrm{K}_{\mathrm{ii}} / \mathrm{p}$. La facilité d'écriture de fonction à variables complexes, la simplicité d'utilisation des tracés de courbes de l'environnement de développement HPVEE 5.0 sont exploitées pour obtenir les courbes de BODE de la boucle non corrigée. Ce support graphique est utilisé pour définir les valeurs de $\mathrm{K}_{\mathrm{ii}}$ et $\mathrm{K}_{\mathrm{pi}}$ suivant des critères de marge de phase ou de bande passante en boucle fermée. Le langage HPVEE 5.0 permet d'écrire, avec une relative facilité, un modèle de simulation de la boucle fermée de courant. Ce modèle de simulation illustre l'influence des valeurs des coefficients du correcteur sur la réponse indicielle.

(d) La dernière séquence de mesures automatiques permet le relevé de la réponse indicielle du courant, le rotor de la machine est bloqué, la consigne est délivrée par le générateur de fonction HP33120A, appliquée sur une entrée analogique du variateur WNTC, le relevé du courant est obtenu sur oscilloscope HP 54600B. Les coefficients du correcteur sont modifiables sur le tableau de bord de la manipulation automatisée. L'objectif de cette séquence est de réaliser une étude critique entre les résultats expérimentaux et ceux obtenus grâce au modèle de simulation.

L'enchaînement des différentes séquences impose une automatisation importante des procédures expérimentales :

- Il est nécessaire de reconfigurer de façon conséquente le variateur WNTC entre les phases successives de manipulations (mode de fonctionnement en asservissement de vitesse, mode de fonctionnement en asservissement de couple). L'informatique le permet en éliminant tout risque d'erreur lié à la modification d'un grand nombre de registres.

- Réaliser des traitements mathématiques sur l'acquisition d'oscillogrammes est chose aisée, 
vue la bibliothèque importante de fonctions disponibles dans l'environnement de développement HPVEE 5.0.

\subsection{Etude de la boucle de vitesse}

Une étude en régime harmonique de l'asservissement de vitesse a pour objectif d'identifier la boucle ouverte de vitesse non corrigée (N302(p)/N307(p)) et de justifier le tracé obtenu de la transmittance de la boucle fermée (N302(p)/N301(p)).

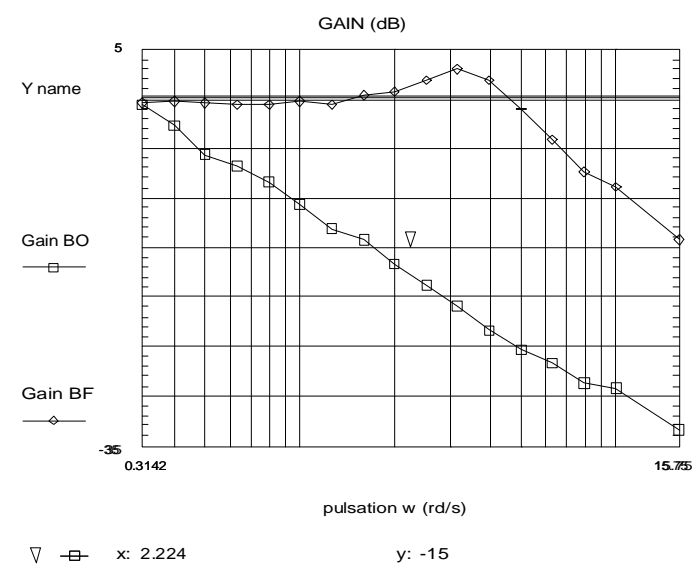

Fig $n^{\circ} 5$ : Courbes de gain, boucle ouverte de vitesse non corrigée, boucle fermée.

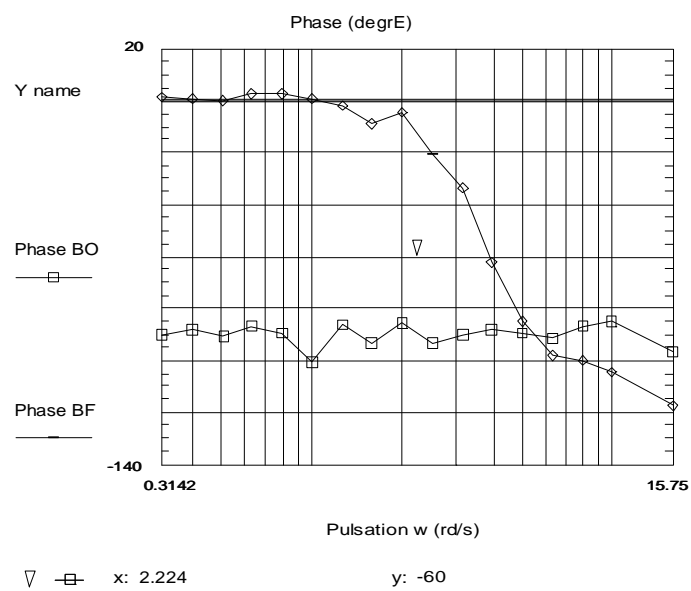

Fig $n^{\circ} 6$ : Courbes de phase, boucle ouverte de vitesse non corrigée, boucle fermée.

Le variateur de vitesse WNTC est configuré afin de rendre disponible le contenu des registres 3.02 et 3.07 sur des sorties de convertisseurs numérique analogique interne à celui-ci. Ces deux signaux et la consigne de vitesse délivrée par le générateur de fonction HP33120A sont envoyés sur trois voies de la centrale d'acquisition HP34970A. Le générateur de fonction est piloté en fréquence mais aussi en amplitude, afin d'éviter la saturation de la boucle de courant pour les points de fréquence plus élevée. La centrale fait l'acquisition des trois signaux, sur une durée de deux périodes, de façon successive, la cohérence temporelle est obtenue grâce à l'horodatage des enregistrements (résolution temporelle 1ms). Les courbes de BODE sont obtenues après extraction du fondamental des enregistrements, par analyse de FOURIER (Certains signaux étant sensiblement pollués). Le tracé des courbes de BODE est reporté aux figures $n^{\circ} 5$ et $n^{\circ} 6$.

Le pilotage automatique de ce type de manipulation est obligatoire :

- Le paramétrage initial du variateur de vitesse et de la centrale d'acquisition demande la modification d'un grand nombre de registres.

- Les traitements mathématiques, pour retrouver la cohérence temporelle puis extraire les fondamentaux des signaux périodiques, sont indispensables pour obtenir des informations amplitude et phase exploitables.

Conclusion : Une expérience de quelques années sur l'utilisation de ce type d'équipement de laboratoire nous confirme que le gain de temps apporté par l'automatisation des procédures de mesures permet aux étudiants de concentrer leurs efforts sur l'interprétation de résultats, sur la validation des modèles d'étude, sur la rédaction des comptes rendus de T.P.. Ces exigences leurs paraissent souvent nouvelles, dans leur scolarité antérieure les activités de travaux pratiques étaient dominées par le relevé manuel de courbes et caractéristiques. Pour autant les activités proposées ne sont pas de type «presse boutons », des compétences de câblage, de paramétrage des instruments de mesure et variateurs de vitesse sont toujours nécessaires et répondent aux exigences attendues dans un premier cycle. Les mesures automatisées ne sont proposées qu'après maîtrise de l'utilisation des instruments et équipements en autonomie. (oscilloscope, variateurs de vitesse etc ...).

Il apparaît évident que l'organisation d'un tel laboratoire d'électrotechnique ne peut être montée sans une coopération interdisciplinaire entre les enseignants de Physique Appliquée et Génie Electrique. L'origine du projet coïncide avec la reconstruction du lycée, le dossier du département électrotechnique n'a pu aboutir sans le soutien de nos Inspecteurs Pédagogiques Régionaux, du Proviseur, du Chef des Travaux du lycée COLBERT, ainsi que du Conseil Régional du Nord Pas de Calais.

\section{Contact : STEVENARD Guy} L.S.T. COLBERT de TOURCOING Parvis Jean Baptiste COLBERT 59200 TOURCOING email : cmrg.stevenard@wanadoo.fr 


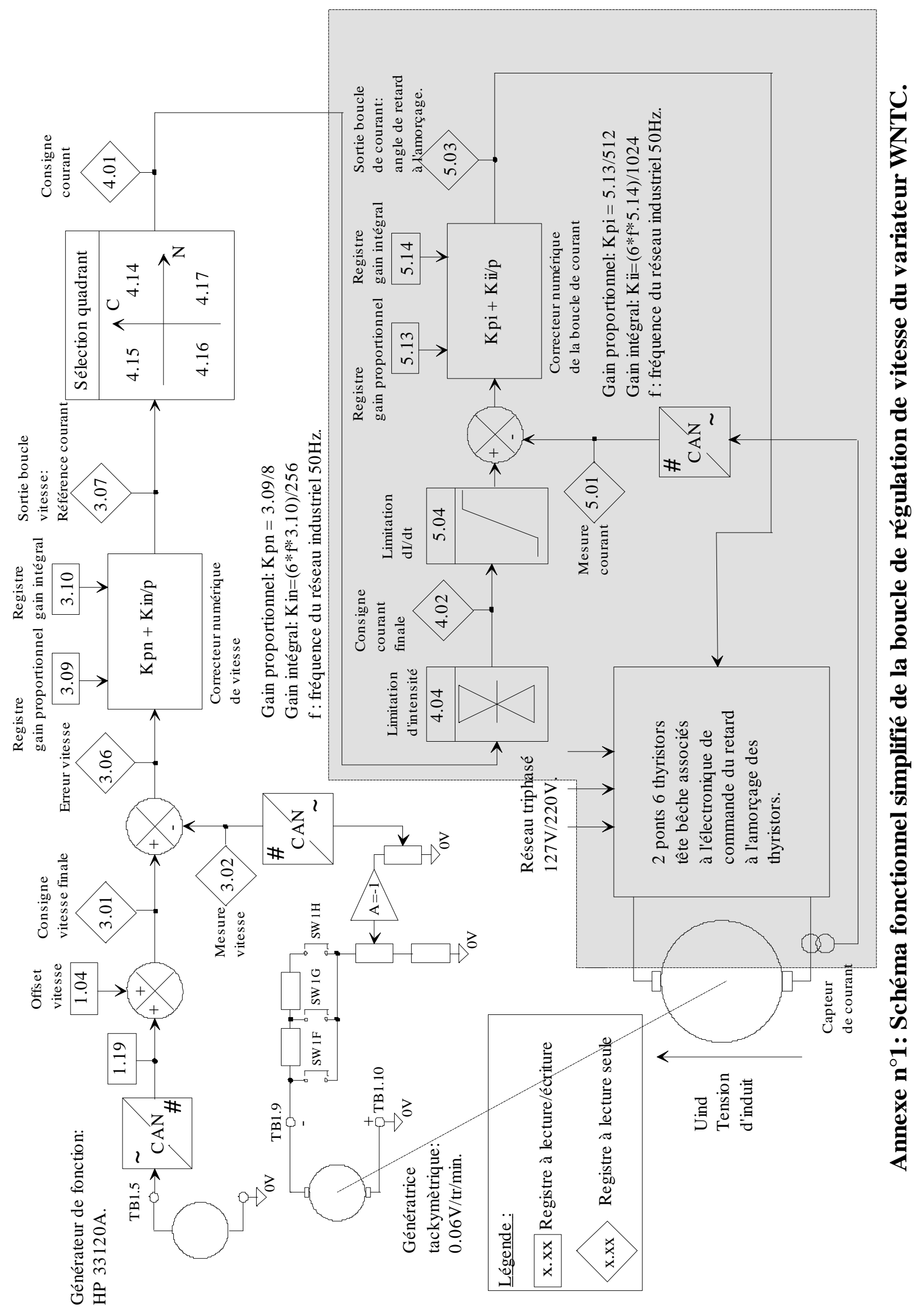

